\title{
An Anti-PM/Scl-75 Antibody-positive Japanese Woman Who Developed Inflammatory Myopathy
}

\author{
Yuki Nakamura ${ }^{1}$, Rei Miyanaga ${ }^{1}$, Hirohiko Shizukawa ${ }^{1}$ and Shun Shimohama ${ }^{2}$
}

\begin{abstract}
:
A 69-year-old Japanese woman presented with mild muscle weakness of the neck and symmetrical proximal parts of the upper and lower limbs. Laboratory tests, needle electromyography, and a muscle biopsy revealed inflammatory myopathy with an apparent clinical classification of polymyositis and positive findings for anti-PM/Scl-75 antibody. This antibody is rare among Japanese populations, and most Japanese patients with the antibody are not classified with the inflammatory myopathy seen in polymyositis. The muscle biopsy also showed marked necrotic and regenerative fibers. We need to collectively investigate patients with the potential to develop this disease, and to identify any unique characteristics for Asian populations, including Japanese.
\end{abstract}

Key words: anti-PM/Scl antibody, inflammatory myopathy, Japanese, muscle biopsy, neurology

(Intern Med 58: 2689-2693, 2019)

(DOI: 10.2169/internalmedicine.2402-18)

\section{Introduction}

Anti-PM/Scl (polymyositis/scleroderma) antibodies (PM/ Scl-75 and PM/Scl-100) have been recognized as a cause of myositis. These antibodies were first detected in patients with an overlapping syndrome of PM and systemic sclerosis (SSc) (1) and have also been recognized in cases of other autoimmune diseases, such as dermatomyositis (DM) (2).

The detection frequency of these antibodies is influenced by the ethnic background, and studies from Europe and North America account for the majority of published data, while studies from Asia remain rare. Some papers have reported anti-PM/Scl antibody-positive DM in Japanese patients (3) and anti-PM/Scl antibody-positive Japanese patients with collagen disease centered on SSc (4). However, a small number of reports have described cases that appear to represent with PM only. No consensus has been reached regarding the clinical characteristics of anti-PM/Scl antibodypositive Japanese patients.

We herein report the case of a Japanese patient with antiPM/Scl-75 antibody-positive inflammatory myopathy in which the clinical classification appeared to be PM and also describe the myopathological findings.

\section{Case Report}

A 69-year-old Japanese woman was referred and admitted to our department with suspected myositis. She had histories of unexplained weight loss over three years and mild muscle weakness from several months before this presentation. She had no history of statin treatment and did not regularly consume alcoholic beverages. No history suggestive of myositis or congenital myopathy was elicited. She had a history of gastroesophageal reflux disease, atrophic gastritis, and glaucoma. A physical examination revealed mild muscle weakness confined to the neck and symmetrical, proximal parts of the upper and lower limbs. The muscles in those areas showed mild atrophy. No skin lesions, such as sclerosis, Gottron's lesion, heliotrope rash, or mechanic's hands, were evident. These skin findings were confirmed by a dermatologist (HT). No myalgia, arthralgia, or dyspnea was observed.

Laboratory tests showed the following: aldolase, $12.7 \mathrm{IU} /$ $\mathrm{L}$; erythrocyte sedimentation rate, $50.9 \mathrm{~mm} / \mathrm{h}$; C-reactive protein, $4.76 \mathrm{mg} / \mathrm{dL}$; creatine kinase $(\mathrm{CK}), 1,081 \mathrm{IU} / \mathrm{L}$; and Krebs von den Lungen (KL)-6, $589 \mathrm{IU} / \mathrm{mL}$ (normal range, <

${ }^{1}$ Department of Neurology, Sapporo Kosei General Hospital, Japan and ${ }^{2}$ Department of Neurology, Sapporo Medical University School of Medicine, Japan

Received: November 19, 2018; Accepted: February 26, 2019; Advance Publication by J-STAGE: June 7, 2019

Correspondence to Dr. Hirohiko Shizukawa, h.shizukawa@ja-hokkaidoukouseiren.or.jp 

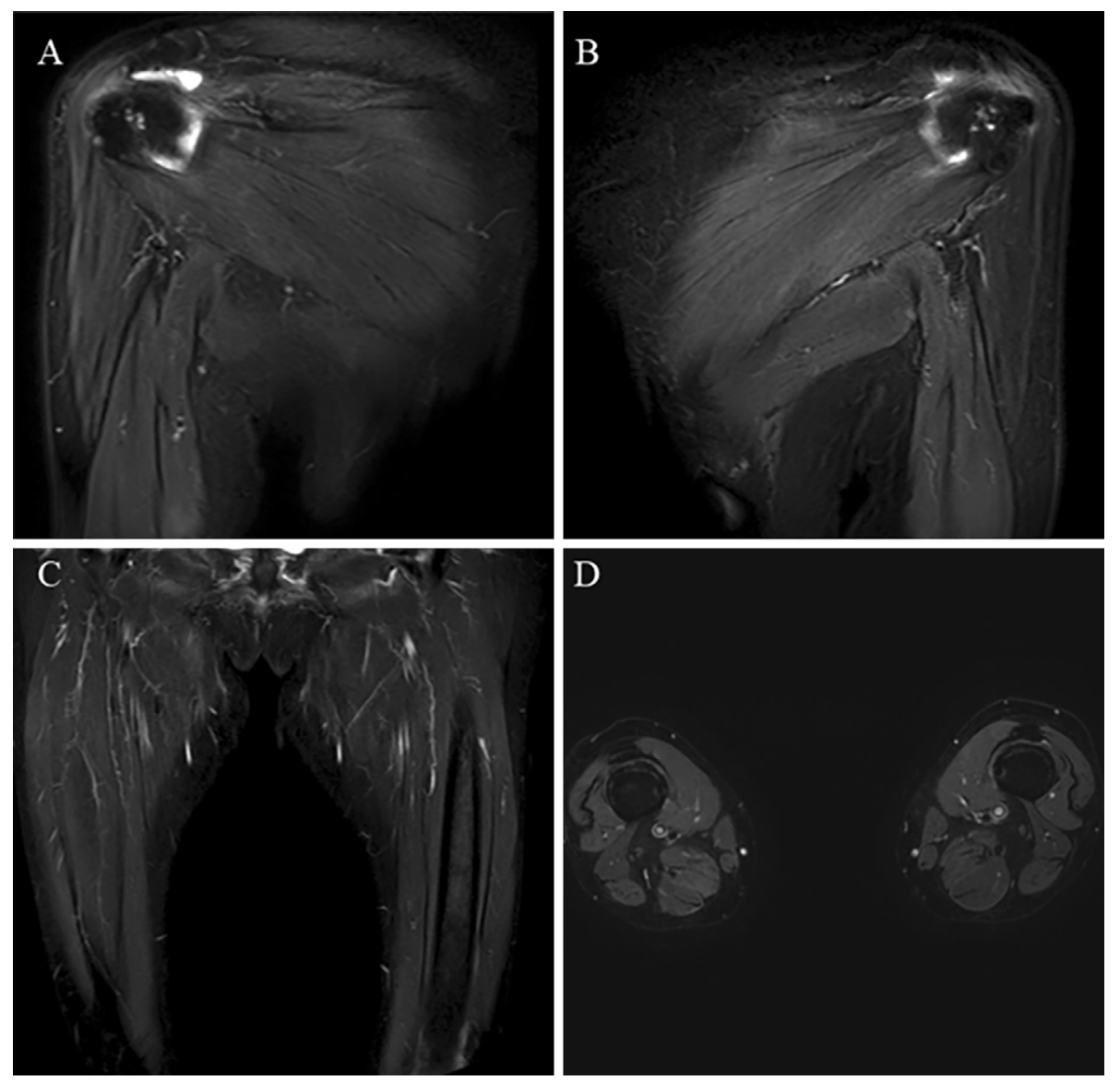

Figure 1. Images from magnetic resonance imaging using short-tau inversion recovery sequencing. Right upper (A), left upper (B), and lower limbs (coronal image: C, axial image: D). No abnormal findings (atrophy, fascial edema, fatty replacement, and muscle edema) are evident.

499 IU/mL). Results for anti-aminoacyl transfer RNA synthetase, anti-DNA, anti-Jo-1, anti-Scl 70, anti-Sjögren's syndrome-A/B, anti-Sm, and anti-U1-ribonucleoprotein were all negative. Antibodies suggesting immune-mediated necrotizing myopathy (anti-signal recognition particle/anti-3hydroxy-3-methylglutaryl coenzyme A reductase) were also negative. Anti-centromere antibody was positive. Anti-PM/ Scl-75 antibody was qualitatively detected using a line blot assay. Negative results were obtained for anti-PM/Scl-100 antibody. Results for molecular human leukocyte antigen (HLA) typing on DNA showed DRB1*01 and DRB1*15.

No abnormalities were evident on magnetic resonance imaging of the upper and lower limbs (Fig. 1). However, needle electromyography of the right upper and lower limbs showed normal spontaneous activity and an abnormal myogenic pattern (early recruitment in voluntary contraction and motor unit potential with small amplitude). A muscle biopsy from the left rectus femoris revealed histological findings reflecting active muscle fiber necrosis and regenerative changes (Fig. 2). Reactive mononuclear cell infiltration was seen surrounding necrotic and regenerating fibers. Immunostaining showed the expression of human leukocyte antigen (HLA)-ABC and deposition of membrane attack complex (MAC) on the muscle fiber membranes. Perifascicular atrophy, fibers with internal nuclei, and endomysial fibrosis were not seen. No perivascular collections of inflammatory cells were apparent. The pathological findings were suggestive of immune-mediated necrotizing myopathy (IMNM), as confirmed by a neurologist specializing in muscle disease (IN). A whole-body search including upper gastrointestinal endoscopy and lower gastrointestinal endoscopy was performed, but no malignant tumors were observed. Chest computed tomography showed mild interstitial changes at the base of the lower lung fields bilaterally.

We initially considered a diagnosis of PM (the PM subset includes IMNM) based on the criteria for idiopathic inflammatory myopathy recently described by Lundberg et al. (5). However, our final diagnosis was inflammatory myopathy with an apparent clinical classification of PM, because the pathological features of anti-PM/Scl antibody-positive myositis have not yet been definitively confirmed, and the pathological features of anti-PM/Scl antibody-positive patients and typical IMNM are not identical (6). Oral prednisolone was initiated at $40 \mathrm{mg} /$ day $(1 \mathrm{mg} / \mathrm{kg} /$ day $)$, and her muscle weakness was gradually improved. After tapering the dose of oral prednisolone, the patient was discharged from the hospital. The serum CK level just before discharge was $211 \mathrm{U} / \mathrm{L}$. As of six months since her discharge, no recurrence has been noted. 

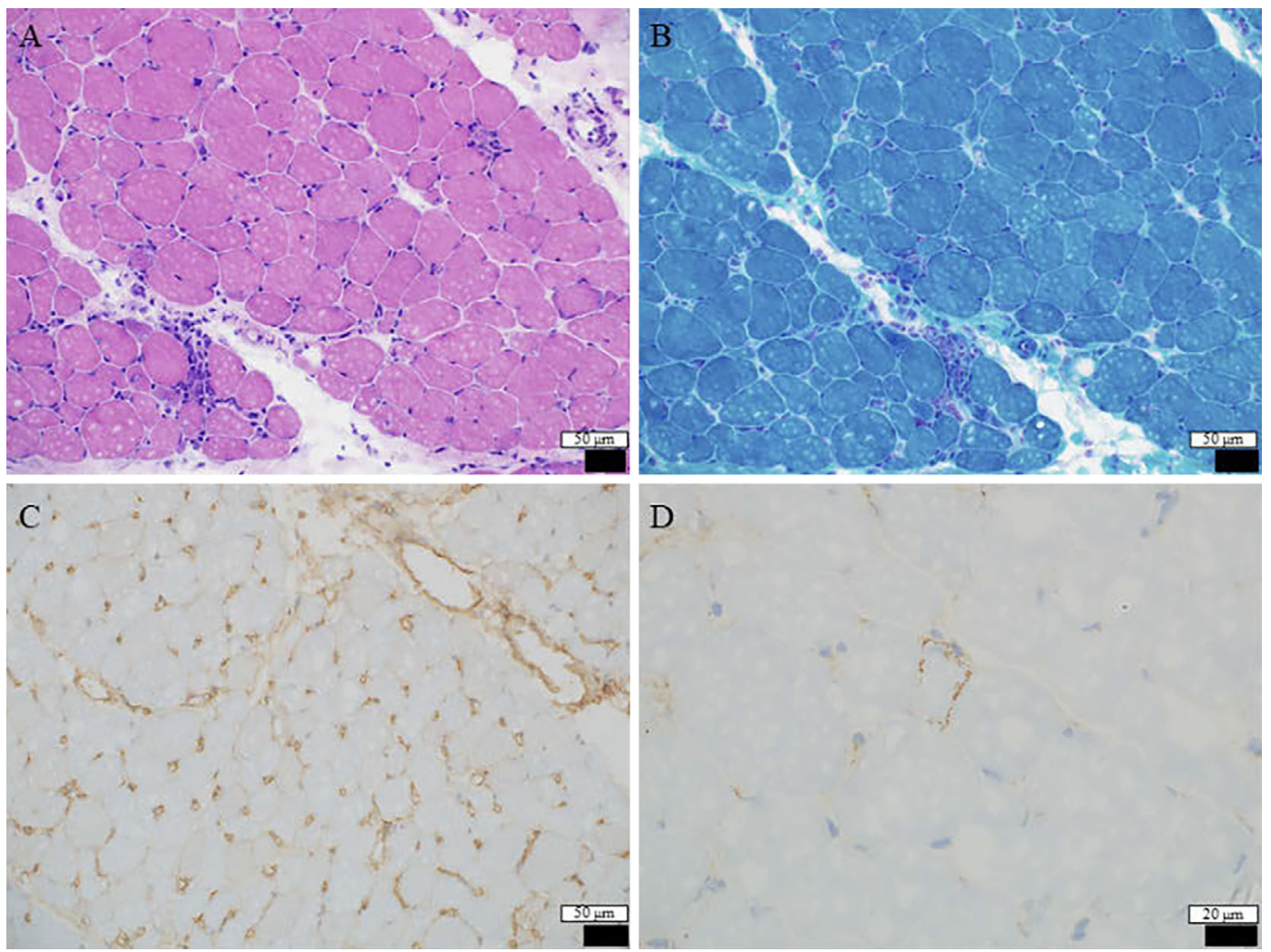

Figure 2. A Hematoxylin and Eosin staining section of the rectus femoris shows mild to moderate variation in the fiber size. A few necrotic fibers and some regenerating fibers are apparent (A). Modified Gomori trichrome-stained sections do not show ragged-red fibers, fibers with rimmed vacuoles, or nemaline rods (B). Immuno-stained sections show the expression of human leukocyte antigen-ABC and deposition of membrane attack complex on the muscle fiber membrane (C, D). Some freezing artifacts are evident.

\section{Discussion}

We encountered a rare case of anti-PM/Scl-75 antibodypositive inflammatory myopathy in a Japanese patient in which the clinical classification appeared closest to be PM. Because the clinical symptoms, such as muscle weakness, were minor and no skin lesions were evident, the clinical findings alone did not indicate such a diagnosis. Laboratory findings, needle electromyography, and a muscle biopsy helped in the diagnosis. The prognosis in this case was favorable.

Previous studies of Japanese individuals have not reported patients diagnosed with inflammatory myopathy, IMNM, and $\mathrm{PM}$ in which anti-PM/Scl antibodies were detected (Table) $(3,4)$. Another two studies to detect anti-PM/Scl antibodies in Japanese patients with systemic autoimmune conditions found no positive cases of anti-PM/Scl antibodies $(7,8)$. For example, in Muro et al., only 9 of 600 patients were ultimately deemed positive for anti-PM/Scl antibodies, indlucing 4 of $16(25 \%)$ patients with undifferentiated connective tissue disease (UCTD), 3 of 126 (2.4\%) patients with DM, 1 of $223(0.4 \%)$ patients with SSc, and 1 of $88(1.1 \%)$ patients with Sjögren's syndrome (4). Two possible reasons for these results were considered. One reason is that anti-PM/Scl antibodies appear to be related to HLADRB1*0301 (9). This HLA type is uncommon in Japanese populations, with an estimated frequency of $0.14 \%$ (4), making larger-scale research difficult. Another reason is that, in order to investigate anti-PM/Scl antibodies, we generally must ask a commercial center in Japan (BML, Tokyo, Japan) to perform testing. Because of the costs incurred, some patients with inflammatory myopathy may not be examined.

Despite the fact that weight loss started over three years earlier, our case was not able to be diagnosed until our department was consulted because of the absence of skin findings and her relatively mild muscle weakness. A small portion of anti-PM/Scl antibody-positive patients develop PM only (10-15), and determining the clinical features in those cases can be difficult. Another study of 41 anti-PM/Sclpositive patients stated that muscle weakness was present in $37 \%$ initially but was ultimately confirmed in $93 \%$ of patients during follow-up (mean follow-up, 6.5 \pm 4.7 years) (6). Long periods from the onset to the recognition of muscle symptoms may be characteristic of anti-PM/Scl-positive patients. Previous series have warned of lung and esophageal involvement (16). We need to perform continuous, long-term observation concerning the existence of new complications such as dysphagia. However, patients with anti-PM/Scl antibody have been considered to show a good prognosis, with 
Table. Comparison of Anti-PM/Scl Antibody-positive Japanese Patients ${ }^{\mathrm{a}}$.

\begin{tabular}{lccccl}
\hline $\begin{array}{c}\text { Patient } \\
\text { Reference) }\end{array}$ & Age & Sex & $\begin{array}{c}\text { PM/Scl-100/ } \\
\text { PM/Scl-75 }\end{array}$ & Diagnosis & \\
\hline A (3) & 64 & M & $+/+$ & DM & Gottron's lesions, facial erythema, IlD, mechanic's hands \\
B (4) & 52 & F & $+/+$ & SS & dry eye, dry mouth \\
C (4) & 62 & F & $+/-$ & SSc & Raynaud's phenomenon, sclerodactyly \\
D (4) & 54 & M & $+/+$ & DM & Gottron's lesions, IlD, mechanic's hands \\
E (4) & 69 & M & $+/+$ & DM & dysphagia, Gottron's lesions, IlD, mechanic's hands, pharyngeal Ca \\
F (4) & 67 & M & $+/+$ & DM & Gottron's lesions, Heliotrope rash, IlD, muscle weakness, prostate Ca \\
G (4) & 73 & F & $+/+$ & UCTD & dry eye, dry mouth, IlD \\
H (4) & 33 & F & $+/-$ & UCTD & morning stiffness, polyarthralgia \\
I (4) & 31 & F & $+/-$ & UCTD & photosensitivity, polyarthralgia \\
J (4) & 31 & F & $+/-$ & UCTD & oral ulcer, photosensitivity \\
K (our case) & 69 & F & $-/+$ & IM & GERD \\
\hline
\end{tabular}

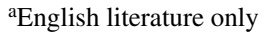

No description of the muscle biopsy was provided for the above-mentioned patients.

Ca: carcinoma, DM: dermatomyositis, F: female, GERD: gastroesophageal reflux disease, IlD: interstitial lung disease, IM: inflammatory myopathy, M: male, PM: polymyositis, SS: Sjögren's syndrome, SSc: systemic scleroderma, UCTD: undifferentiated connective tissue disease

a good response to steroids and relatively low rate of malignancy $(6,15)$. Our case also responded well to oral prednisolone, and no cancer was found.

The findings from the muscle biopsy were a point of interest in the present study. To our knowledge, there have been no reports of the muscle biopsy findings for anti-PM/ Scl antibody-positive patients in Asian populations. Previous studies concerning the clinicoserologic and pathologic classifications of inflammatory myopathies have shown that 10 of 178 patients had anti-PM/Scl antibodies, with 9 of those 10 patients $(90 \%)$ presenting with necrotic and regenerative fibers and 9 of the 10 patients $(90 \%)$ presenting with the expression of major histocompatibility complex, class 1 (only sparse fibers or groups of fibers in 1 patient, on the surface of most/all fibers in 3 patients, and perifascicular or diffuse with reinforcement in the perifascicular region in 6 patients) (17). These features were consistent with the findings in our case. However, another study of four anti-PM/Scl antibody-positive cases suggested that fibrosis was significantly associated with anti-PM/Scl antibodies (18). A recently published paper showed that perivascular inflammation was the most commonly observed feature among anti$\mathrm{PM} / \mathrm{Scl}$ antibody-positive patients (6). Different histological features have been reported in the literature, and the pathological findings of anti-PM/Scl antibody-positive myositis have yet to be definitively established. Applying the current classification criteria to our case was difficult, and we diagnosed the patient with inflammatory myopathy. The present report was from an area in which the onset of anti-PM/Scl antibody-associated disease is rare. Further cases need to be accumulated and examined not only from a serological perspective but also from a pathological point of view.

Several limitations associated with the present study warrant mention. Testing for anti-PM/Scl-75 antibody was performed by a commercial center in Japan. The antibody titer was not known because the results were qualitative in nature. The reliability of measurements performed by external companies is not necessarily high, but the same measurement method has also been used in recently published research (6).

In conclusion, we encountered an anti-PM/Scl-75 antibody-positive Japanese patient who showed inflammatory myopathy with an apparent clinical classification of PM. We reached the eventual diagnosis using clinical findings, laboratory tests, needle electromyography, and a muscle biopsy. Physicians should be aware that patients with anti-PM/Scl antibodies may be present among those with suspected myositis who have not yet received a confirmed diagnosis.

The authors state that they have no Conflict of Interest (COI).

\section{Acknowledgement}

We wish to thank Hiroyuki Takahashi, MD, PhD (skin findings and the muscle biopsy) and Ichizo Nishino, MD, PhD (analysis and interpretation of the muscle biopsy).

\section{References}

1. Wolfe JF, Adelstein E, Sharp GC. Antinuclear antibody with distinct specificity for polymyositis. J Clin Invest 59: 176-178, 1977.

2. D'Aoust J, Hudson M, Tatibouet S, et al. Clinical and serologic correlates of anti-PM/Scl antibodies in systemic sclerosis: a multicenter study of 763 patients. Arthritis Rheumatol 66: 1608-1615, 2014.

3. Kohara A, Yanaba K, Muro Y, et al. Anti-PM/Scl antibody-positive dermatomyositis in a Japanese patient: a case report and review of the literature. Int J Rheum 20: 2186-2189, 2017.

4. Muro Y, Hosono Y, Sugiura K, Ogawa Y, Mimori T, Akiyama M. Anti-PM/Scl antibodies are found in Japanese patients with various systemic autoimmune conditions besides myositis and scleroderma. Arthritis Res Ther 17: 57, 2015.

5. Lundberg IE, Tjärnlund $A$, Bottai M, et al. 2017 European League Against Rheumatism/American College of Rheumatology classification criteria for adult and juvenile idiopathic inflammatory myopathies and their major subgroups. Ann Rheum Dis 76: 19551964, 2017. 
6. De Lorenzo R, Pinal-Fernandez I, Huang W, et al. Muscular and extramuscular clinical features of patients with anti-PM/Scl autoantibodies. Nsurology 90: e2068-e2076, 2018.

7. Hirakata M, Mimori T, Akizuki M, Craft J, Hardin JA, Homma M. Autoantibodies to small nuclear and cytoplasmic ribonucleoproteins in Japanese patients with inflammatory muscle disease. Arthritis Rheum 35: 449-456, 1992.

8. Kaji K, Fertig N, Medsger TA Jr, et al. Autoantibodies to RuvBL1 and RuvBL2: a novel systemic sclerosis-related antibody associated with diffuse cutaneous and skeletal muscle involvement. Arthritis Care Res (Hoboken) 66: 575-584, 2014.

9. Hausmanowa-Petrusewicz I, Kowalska-Oledzka E, Miller FW, et al. Clinical, serologic, and immunogenetic features in Polish patients with idiopathic inflammatory myopathies. Arthritis Rheum 40: 1257-1266, 1997

10. Reichlin M, Maddison PJ, Targoff I, et al. Antibodies to a nuclear/ nucleolar antigen in patients with polymyositis overlap syndromes. J Clin Immunol 4: 40-44, 1984.

11. Brouwer R, Hengstman GJ, Vree Egberts W, et al. Autoantibody profiles in the sera of European patients with myositis. Ann Rheum Dis 60: 116-123, 2001.

12. Selva-O'Callaghan A, Labrador-Horrillo $M$, Solans-Laque $R$, Simeon-Aznar CP, Martínez-Gómez X, Vilardell-Tarrés M. Myositis-specific and myositis-associated antibodies in a series of eighty-eight Mediterranean patients with idiopathic inflammatory myopathy. Arthritis Rheum 55: 791-798, 2006.
13. Mahler M, Raijmakers R, Dähnrich $C$, Blüthner $M$, Fritzler MJ. Clinical evaluation of autoantibodies to a novel $\mathrm{PM} / \mathrm{Scl}$ peptide antigen. Arthritis Res Ther 7: R704-R713, 2005.

14. Rozman B, Cucnik S, Sodin-Semrl S, et al. Prevalence and clinical associations of anti-Ku antibodies in patients with systemic sclerosis: a European EUSTAR-initiated multi-centre case-control study. Ann Rheum Dis 67: 1282-1286, 2008.

15. Vandergheynst F, Ocmant A, Sordet C, et al. Anti-pm/scl antibodies in connective tissue disease: clinical and biological assessment of 14 patients. Clin Exp Rheumatol 24: 129-133, 2006.

16. Marie I, Lahaxe L, Benveniste $\mathrm{O}$, et al. Long-term outcome of patients with polymyositis/ dermatomyositis and anti-PM-Scl antibody. Br J Dermatol 162: 337-344, 2010.

17. Fernandez C, Bardin N, De Paula AM, et al. Correlation of clinicoserologic and pathologic classifications of inflammatory myopathies: study of 178 cases and guidelines for diagnosis. Medicine (Baltimore) 92: 15-24, 2013.

18. Paik JJ, Wigley FM, Lloyd TE, et al. Spectrum of muscle histopathologic findings in forty-two scleroderma patients with weakness. Arthritis Care Res (Hoboken) 67: 1416-1425, 2016.

The Internal Medicine is an Open Access journal distributed under the Creative Commons Attribution-NonCommercial-NoDerivatives 4.0 International License. To view the details of this license, please visit (https://creativecommons.org/licenses/ by-nc-nd/4.0/).

(C) 2019 The Japanese Society of Internal Medicine Intern Med 58: 2689-2693, 2019 